\title{
Characterization of Adult Transcriptomes from the Omnivorous Lady Beetle Coleomegilla maculata Fed Pollen or Insect Egg Diet
}

\author{
Margaret Louise Allen $\bowtie$ \\ US Department of Agriculture, Agricultural Research Service, Biological Control of Pests Research Unit, USA.
}

$\triangle$ Corresponding author: Office Telephone 662-686-3647 Fax 662-686-5281 Email meg.allen@ars.usda.gov.

(C) Ivyspring International Publisher. This is an open-access article distributed under the terms of the Creative Commons License (http:/ / creativecommons.org/ licenses/by-nc-nd/3.0/). Reproduction is permitted for personal, noncommercial use, provided that the article is in whole, unmodified, and properly cited.

Published: 2015.01.10

\begin{abstract}
Diet, nutrition, and obesity are important topics of current research. While many insect genome and/or transcriptome models are based on dietary specialists, the lady beetle Coleomegilla maculata, a common New World species, is highly omnivorous. C. maculata feeds on plants, fungi, insects and other arthropods; its diet frequently includes conspecific cannibalism. This study reports and discusses the first nutritionally based $C$. maculata transcriptomes. These transcriptomes were prepared from highly inbred specimens provided limited diets, after adult eclosion, of either pollen only or eggs of a soft bodied hemipteran insect only. Selected sequences from the transcriptomes were compared to verify basic genetic similarity of the sampled individuals. Differentially expressed genes associated with these diets were identified to aid with studies of omnivore diet and nutrition. Selected transcriptome sequences described herein are filed with the National Center for Biotechnology Information (NCBI), GenBank Bioproject PRJNA236444.
\end{abstract}

Key words: insect nutrition, omnivory, digestion, RNA stability, gene expression, Coccinellidae, biological control.

\section{Introduction}

North American agroecosystems are highly manipulated, particularly in the United States. Nonetheless, certain key insects persist within these ecosystem, acting as pests (consumers of or damaging to crops), as commensals, and as beneficials. One key beneficial insect found in many U. S. agroecosystems is the lady beetle Coleomegilla maculata (Coccinellidae: Coleoptera). C. maculata is a widely distributed [1] native North American species complex [2] and is generally accepted as an ecological indicator [3] and used in agricultural and ecological research as a representative non-target organism [4, 5]. Given its importance in agricultural ecology, the biology of $C$. maculata has been well studied. Studies of the diet of C. maculata have evaluated nutrition, focusing on pollen [6, 7], prey [8], and aiming at artificial diet development [9]. In the present work, gene expression in otherwise identical inbred specimens of this species of lady beetle are compared after restricted dietary intake, during the imaginal (adult) stage, of either pollen or insect eggs. The genetic resources described herein will facilitate further research on diet and digestive function in omnivorous organisms.

\section{Results and Discussion}

While C. maculata is accepted as an ecologically important lady beetle, it has not been widely utilized as a biological control agent. Some species of the family Coccinellidae are used as biological control in the US, such as Hippodamia convergens and Adalia bipunc- 
tata [10]. Harmonia axiridis, an introduced but invasive lady beetle in the US, has recently been modified to produce transgenic research organisms, and will be useful as a genetic model [11]. C. maculata has characters that make it a preferred model, including ease of maintenance [12] and visible phenotypic mutant strains ([13] and unpublished results). The genome size of $C$. maculata is relatively small, estimated at 0.19 pg [14]; based on standard conversion, assuming a diploid genome:

\section{Genome size $(\mathrm{Mbp})=978 \times$ DNA content $(\mathrm{pg})$}

this is roughly equivalent to $186 \mathrm{Mbp}$ [15] so sequencing the full genome of this insect should be achieveable. A reference transcriptome of C. maculata has been published to the internet (http://2ei.univ-perp.fr/?page_id=89, accessed 22 July 2014). The transcripts provided by the study described herein will facilitate further molecular genetic, biochemical, and physiological investigation. Because the insects used for this study were highly inbred and also siblings, the sequences represent a limited number of alleles, and do not represent the wider populations of the species in Mississippi or the broader environment. On the other hand, the sequences obtained may be used for a baseline to determine population genetics and identify variability in the species and between subspecies.

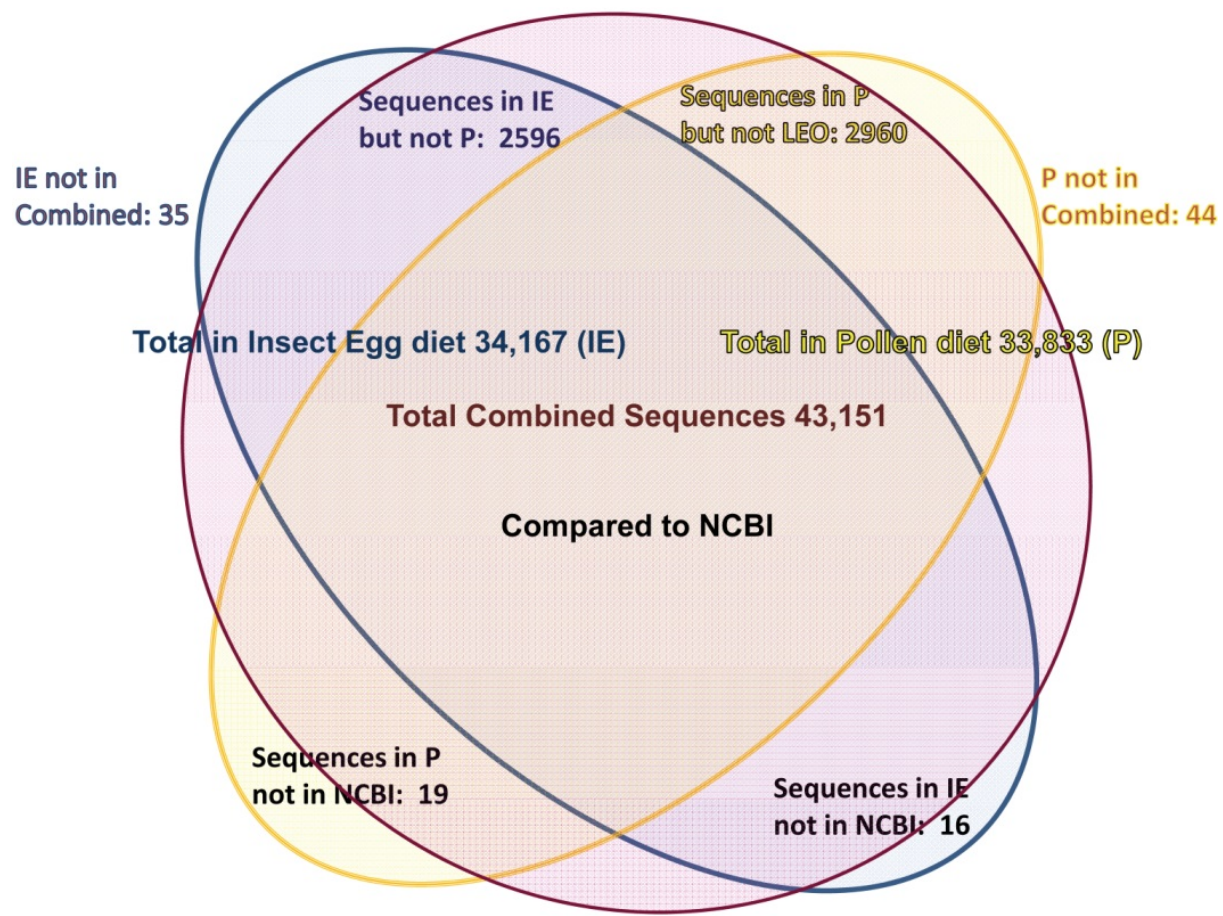

Figure 1. Diagram of comparison of total contiguous sequences generated from adult insect samples fed either a diet of only insect eggs (IE) or only pollen (P).
Assemblies of the total RNA yielded 33,833 assembled sequences from the pollen fed treatment and 34,167 assembled sequences from the insect egg fed treatment. The combined sequences assembled into 43,151 sequences. Average sequence lengths were 1403, 1300, and 1456 respectively. While many of the treatment group sequences were identical or nearly so, there were representatives of unique sequences as shown in Figure 1. Because the assemblies were de novo, many unique and unclassified sequences were expected. To gain an insight on the similarities between the assemblies, sequences were analyzed by NCBI BLAST® (US National Library of Medicine) [16] using the tBLASTx "search translated nucleotide database using a translated nucleotide query" (database accessed 22 May 2013). The resulting BLAST spreadsheets were sorted and the longer and more similar (to NCBI accessions) sequences were examined. Sequences shorter than $500 \mathrm{nt}$ and with expect values $>1$ e-10 were discarded. The remaining sequences amounted to around $10 \%$ of the total assembled sequences: 3,376 from the pollen fed treatment, and 3,358 from the insect egg fed treatment (Table 1A, Table 1B). The sequences from both treatments were primarily similar to other insect sequences $(95 \%$, +/$0.5 \%$ ), and to RNA sequences (not specifically analyzed). The largest portion of similarities by insect order was to Coleoptera, as expected (49\%), as shown by two pie charts, one for each treatment, in Figure 2. The individual insects used for sequencing were inbred siblings, thus many identical sequences from the two samples were expected. The greatest number of similar sequences in GenBank came from the two beetle species Tribolium castaneum and Dendroctonus ponderosae. To validate the baseline similarity of our sequence sets, sixteen sequences that were most closely related to the same GenBank sequence, and at similar expect values and similar lengths, were chosen from each treatment and compared pairwise in BLAST (BLAST two sequences option). Results are shown in Table 2; all comparisons were at least $99 \%$ identical at the nucleotide level. These results support the assumption that 
the two sets of sequences represent nearly identical specimens, differing primarily in those transcripts of genes responding to diet provided to the adult insects. Also as expected, some sequences that were unique to the treatments were associated with the diet [17]. Among the pollen fed sequences were hits similar to plant sequences, and among the egg fed sequences were hits similar to the genus of the insect eggs, Lygus spp. Interestingly, some of the egg diet sequences were nearly identical (e-value 0 ) to a virus recently described from Lygus lineolaris [18]. It appears that the viable Lygus spp. eggs used as diet were carrying the virus, indicating that the virus was present in the laboratory colony and was able to resist degradation by the digestive system of $C$. maculata. The implications of this finding may be important for future pest control strategies aimed to utilize genetically modified or pathogenic viruses.

\section{Insect egg (IE) diet treatment: sequences with closest simlarity to NCBI accessions (score E-10 or better)}

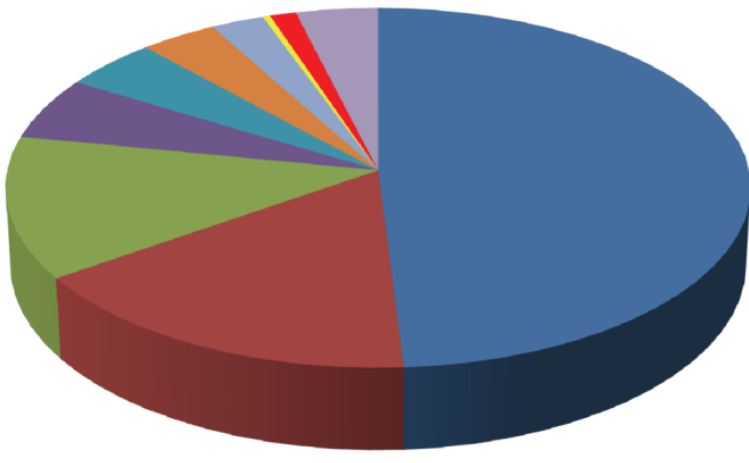

- Coleoptera $49 \%$

- Hymenoptera 16\%

- Neuroptera 13\%

- Diptera 6\%

- Pthiraptera 4\%

- Lepidoptera 3\%

- Hemiptera 3\%

other insect orders $0 \%$

microbial $1 \%$

non-insect metazoan $4 \%$

Pollen (P) diet: sequences with closest simlarity to NCBI accessions (score $\mathrm{E}-10$ or better)

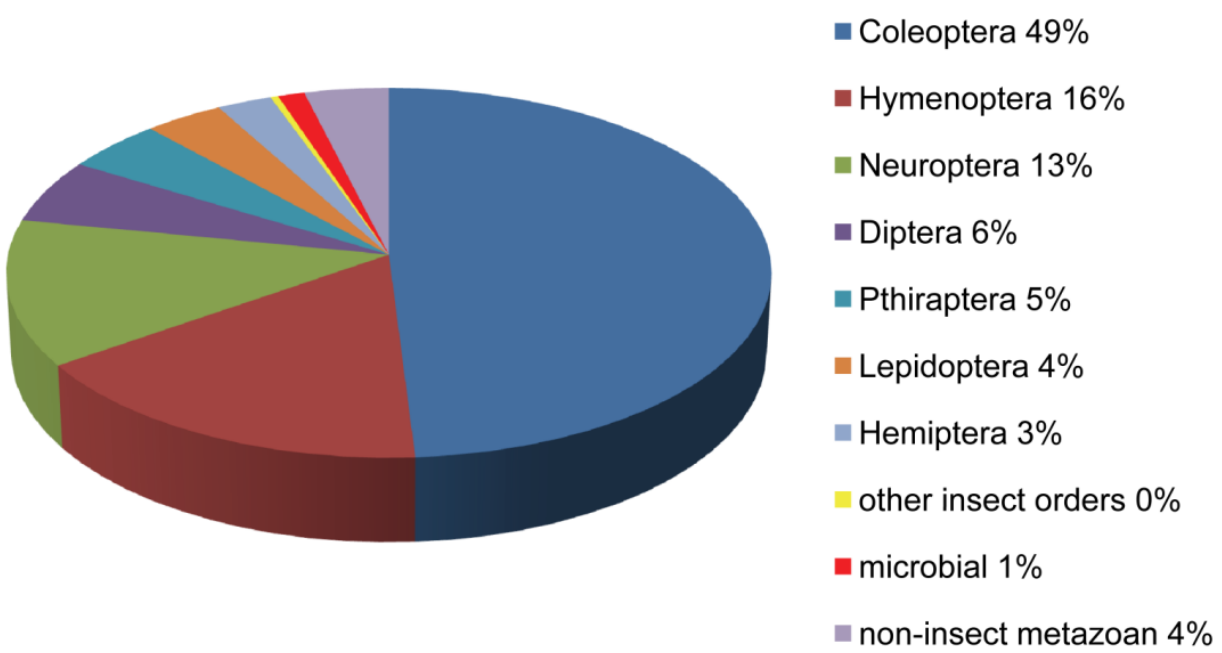

Figure 2. Individual transcriptomes of samples are very similar in overall characteristics, as expected for inbred sibling samples. Pie chart comparison of most similar (expect <e-10) and longest (>500 nt) contiguous sequences generated from adult insect samples fed either a diet of only insect eggs (IE) or only pollen $(\mathrm{P})$. Roughly $10 \%$ of the total sequences are represented. 
Table 1A. Closest homologous sequences; quantities by taxonomy. Treatment: pollen diet, 3376 non-redundant sequences with expect value of $<1.00 \mathrm{e}-10$. Right hand columns are insect genera, left hand columns are non-insect. Sequences that differ from the insect egg diet treatment in quantity of hits are indicated by asterisk $(*)$.

$\begin{array}{ll} & \text { Non-insect } \\ \dagger & \text { Genus } \\ * & \text { virus } \\ & \text { Phyllobacterium } \\ & \text { Sinorhizobium } \\ & \text { Crinalium } \\ & \text { Cronobacter } \\ & \text { uncultured bacteria } \\ & \text { Dictyostelium } \\ & \text { Entamoeba } \\ * & \text { Babesia } \\ * & \text { Neospora } \\ * & \text { Candida } \\ & \text { Kluyveromyces } \\ * & \text { Leptosphaeria } \\ & \text { Millerozyma } \\ * & \text { Rhizopus } \\ * & \text { Tetrapisispora } \\ & \text { Naegleria } \\ & \text { Hydra } \\ * & \text { Caenorhabditis } \\ & \text { Ditylenchus } \\ & \text { Haemonchus } \\ & \text { Trichinella } \\ & \text { Trichoplax } \\ * & \text { Dugesia } \\ & \text { Schiostosoma } \\ * & \text { Trichobilharzia } \\ * & \text { uncultured eukaryote } \\ & \\ & \text { Total } \\ & \\ & \end{array}$

Non-insect Genus Platynereis Urechis

* Amblyomma

* Ixodes

Metaseiulus

Phrynus

Latrodectus

* Lepeophtheirus Tachypleus Branchiostoma

Ichthyophthiriu

Nematostella

* Strongylocentrotus

Saccoglossus

Chlamys

* Sepia

* Bithynia

* Plakortis

Amphimedon

Suberites

Ciona

Rana

* Xenopus

Meleagris

Taeniopygia

Discopyge

Bos

Canis

Cavia

Cricetulus

Dasypus

* Loxodonta

Macaca

Monodelphis

Mus

Nomascus

Ochotona

Odobenus

Orcinus

* Ornithorhynchus

* Otolemur

Rattus

* Saimiri

Sarcophilus

Trichechus

Anolis

* Charina

Chrysemys

Gallus $\begin{array}{cl}\text { hits } & \\ 5 & \text { virus } \\ 2 & \text { Bacteri }\end{array}$

Bacteria

1 Bacteria

1 Bacteria

1 Bacteria

1 Eukaryota

Eukaryota

Eukaryota Apicomplexa

Eukaryota Fungi

Eukaryota Fungi

1 Eukaryota Fungi

Eukaryota Fungi

Eukaryota Fungi

Eukaryota Fungi

Eukaryota Heterolobosea

3 Eukaryota Hydrozoa

4 Eukaryota Nematoda

1 Eukaryota Nematoda

Eukaryota Nematoda

Eukaryota Nematoda

2 Eukaryota Placozoa

2 Eukaryota Platyhelminthes

Eukaryota Platyhelminthes

Eukaryota Platyhelninthes

Eukaryota unknown

Non-specific higher taxonomic grouping, common name Annelida Polychigh Annelida Polychaeta

Arthropoda Acari

Arthropoda Acari

Arthropoda Acari

Arthropoda Arachnida

Arthropoda Araneae

Arthropoda Copepoda

Arthropoda Limulidae

Cephalochor Branchiostomidae

Ciliophora Oligohymenophorea

Cnidaria Actiniaria

Echinoderma Euechinoidea

Hemichordat Harrimaniidae

1 Mollusca Bivalvia

1 Mollusca Decapodiformes

Mollusca Gastropoda

1. Porifera Homoscleromorpha

Porifera

1 Porifera Tetractinomorpha

Tunicata Enterogona

Vertebrata Amphibia

Vertebrata Amphibia

Vertebrata Aves

Vertebrata Aves

Vertebrata Chondrichthyes

Vertebrata Mammalia

Vertebrata Mammalia

Vertebrata Mammalia

1 Vertebrata Mammalia

1 Vertebrata Mammalia

Vertebrata Mammalia

1 Vertebrata Mammalia

Vertebrata Mammalia

Vertebrata Mammalia

Vertebrata Mammalia

2 Vertebrata Mammalia

1 Vertebrata Mammalia

Vertebrata Mammalia

Vertebrata Mammalia

8 Vertebrata Mammalia

1. Vertebrata Mammalia

1 Vertebrata Mammalia

2 Vertebrata Mammalia

2 Vertebrata Mammalia

3 Vertebrata Mammalia

1 Vertebrata Percomorpharia

4 Vertebrata Sauria

Vertebrata Sauria

1 Vertebrata Sauria

1 Vertebrata Sauria

(continued from left)

$\begin{array}{lcllrl}\text { Insect Genus } & \text { hits } & \text { Order } & \text { Family } & \text { Order total } & \text { Percent } \\ \text { Periplaneta } & 1 & \text { Blattodea } & \text { Blattidae } & & \\ \text { * Blattella } & 2 & \text { Blattodea } & \text { Ectobiidae } & & \\ \text { Biphyllus } & 3 & \text { Coleoptera } & \text { Biphyllidae } & 1664 & 49.29 \% \\ \text { * Dastarcus } & 1 & \text { Coleoptera } & \text { Bothrideridae } & & \end{array}$

* Dastarcus $\quad 1$ Coleoptera Bothrideridae

Cerambicidae

Cerambicidae

Chrysomelidae

Chrysomelidae

Chrysomelidae

Chrysomelidae

Chrysomelidae

Chrysomelidae

Chrysomelidae

Chrysomelidae

Coccinellidae

Coccinellidae

Coccinellidae

Coccinellidae

Coccinellidae

Coccinellidae

Coccinellidae

Coccinellidae

Curculionidae

Curculionidae

Curculionidae

Curculionidae

Curculionidae

Curculionidae

Curculionidae

Dytiscidae

Dytiscidae

Elateridae

Elateridae

Georissidae

Histeridae

Meloidae

Mycetophagidae

Scarabaeidae

Scarabaeidae

Tenebrionidae

Tenebrionidae

Tenebrionidae

Conopidae

Culicidae

Culicidae

Culicidae

Culicidae

Diopsidae

Drosophilidae

Hybotidae

Muscidae

Psychodidae

Stratiomyidae

Tephritidae

Tephritidae

Tephritidae

Tipulidae

Aeyrodidae

Alydidae

Aphididae

Aphidide

Aphidide

Belostomatidae

Delphacidae

Pseudococcidae

Primate

Didelphidae (oppossum)

Rodentia (mouse)

Primate (gibbon)

pika

Odobenidae (walrus)

Delphinidae (dolphins)

Monotremata (platypus)

Rodentia (rat)

squirrel monkey

Dasyuridae (Tasmanian devil)

Trichechidae (manatee)

Iguanidae

Serpentes (snakes)

Testudines

Phasianidae (fowl)
Notonectidae

Psyllidae

2 Hemiptera

2 Hemiptera

1 Hemiptera

224 Hymenoptera

6 Hymenoptera Apidae

1 Hymenoptera Braconidae

7 Hymenoptera Braconidae

63 Hymenoptera Braconidae

1 Hymenoptera Formicidae

76 Hymenoptera Megachilida

47 Lepidoptera Bombycidae

1 Lepidoptera

7 Lepidoptera

Lepidopter

Lepidopt

1 Lepidoptera

1 Lepidoptera

Bombycid
Cossidae

Crambidae

Geometridae

Lecithoceridae

Limacodidae

Noctuidae
84 Hymenoptera Megachilidae

Lasiocampidae

$190 \quad 5.63 \%$

$86 \quad 2.55 \%$

$552 \quad 16.35 \%$

$124 \quad 3.67 \%$ 


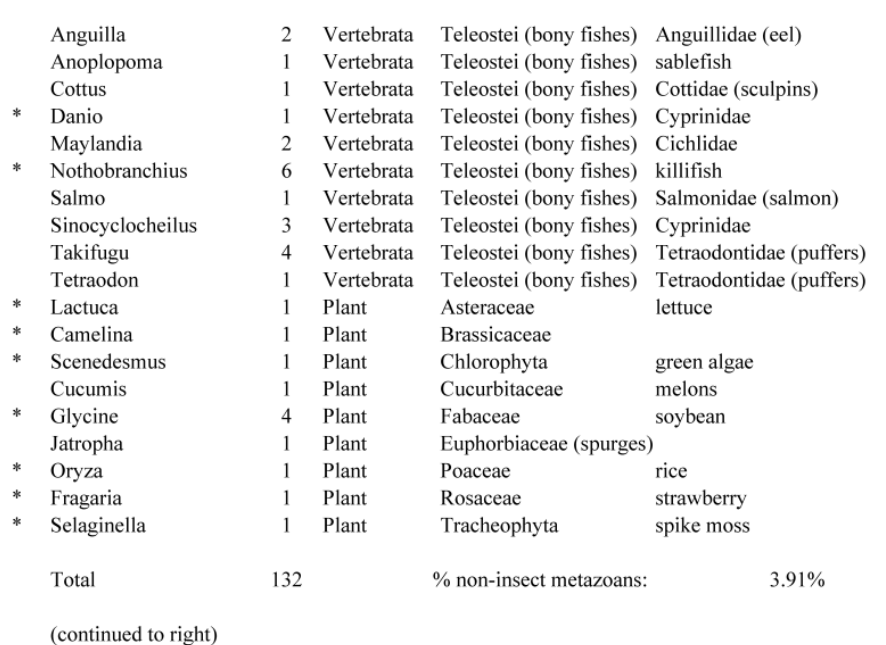

$\mid \begin{array}{lcl}* \text { Heliothis } & 1 & \text { Lepidoptera } \\ \text { Spodoptera } & 25 & \text { Lepidoptera } \\ \text { Euphydryas } & 2 & \text { Lepidoptera } \\ { }^{*} \text { Heliconius } & 12 & \text { Lepidoptera } \\ \text { Poladryas } & 1 & \text { Lepidoptera } \\ \text { * Papilio } & 11 & \text { Lepidoptera } \\ \text { Antheraea } & 1 & \text { Lepidoptera } \\ \text { Manduca } & 1 & \text { Lepidoptera } \\ \text { * Monochamus } & 4 & \text { Lepidoptera } \\ \text { Neococytius } & 1 & \text { Lepidoptera } \\ \text { Edosa } & 1 & \text { Lepidoptera } \\ \text { Leucomele } & 1 & \text { Lepidoptera } \\ \text { Tinea } & 1 & \text { Lepidoptera } \\ \text { * Argyresthia } & 1 & \text { Lepidoptera } \\ \text { * Zelleria } & 1 & \text { Lepidoptera } \\ \text { * Chrysopa } & 431 & \text { Neuroptera } \\ \text { * Locusta } & 2 & \text { Orthoptera } \\ \text { * Schistocerca } & 1 & \text { Orthoptera } \\ \text { Dianemobius } & 1 & \text { Orthoptera } \\ * \text { Gryllus } & 3 & \text { Orthoptera } \\ { }^{*} \text { Pediculus } & 154 & \text { Pthiraptera } \\ \text { Xenopsylla } & 1 & \text { Siphonaptera } \\ \text { Dipseudopsis } & 1 & \text { Trichoptera } \\ & & \\ \text { Total insect hits } & 3213 & \\ & & \end{array}$

Noctuidae

Noctuidae

Nymphalidae

Nymphalidae

Nymphalidae

Papilionidae

Saturniidae

Sphingidae

Sphingidae

Sphingidae

Tineidae

Tineidae

Tineidae

Yponomeutidae

Yponomeutidae

Chrysopidae

$431 \quad 12.77 \%$

Acrididae

Acrididae

Gryllidae

Gryllidae

Pediculidae

Pulicidae

Dipseudopsidae

\begin{tabular}{rr}
154 & $4.56 \%$ \\
12 & $0.36 \%$ \\
\hline 3213 & $95.17 \%$
\end{tabular}

Table 1B. Closest homologous sequences; quantities by taxonomy. Treatment: insect egg diet, 3358 non-redundant sequences with expect value of $<1.00 \mathrm{e}-10$. Right hand columns are insect genera, left hand columns are non-insect. Sequences that differ from the pollen diet treatment in quantity of hits are indicated by asterisk $(*)$.

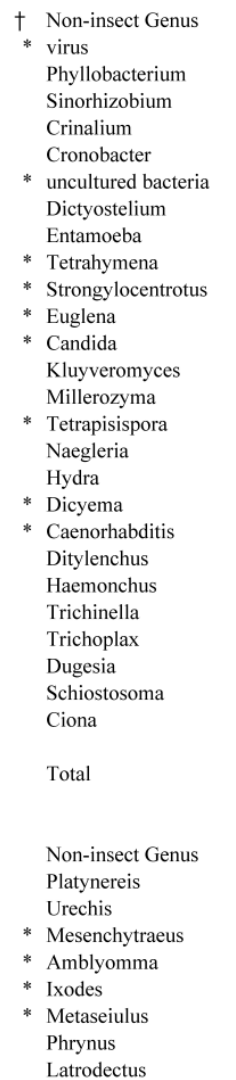

\begin{tabular}{ll}
6 & virus \\
2 & Bacteria \\
1 & Bacteria \\
1 & Bacteria \\
1 & Bacteria \\
1 & Bacteria \\
1 & Eukaryota \\
1 & Eukaryota \\
1 & Eukaryota \\
4 & Eukaryota \\
1 & Eukaryota \\
3 & Eukaryota \\
1 & Eukaryota \\
1 & Eukaryota \\
2 & Eukaryota \\
1 & Eukaryota \\
3 & Eukaryota \\
1 & Eukaryota \\
6 & Eukaryota \\
1 & Eukaryota \\
1 & Eukaryota \\
1 & Eukaryota \\
2 & Eukaryota \\
1 & Eukaryota \\
2 & Eukaryota \\
1 & Eukaryota \\
\hline &
\end{tabular}

47

$\begin{array}{ll}1 & \text { Annelida } \\ 1 & \text { Annelida } \\ 1 & \text { Annelida } \\ 1 & \text { Arthropoda } \\ 7 & \text { Arthropoda } \\ 3 & \text { Arthropoda } \\ 1 & \text { Arthropoda } \\ 1 & \text { Arthropoda }\end{array}$

\begin{tabular}{ll}
\multicolumn{2}{l}{ Non-specific higher taxonomic grouping } \\
Alphaproteobacteria & Rhizobiales \\
$\begin{array}{l}\text { Alphaproteobacteria } \\
\text { Cyanobacteria } \\
\text { Gammaproteobacteria }\end{array}$ & Rhizobiales \\
unknown & Enterobacteriaceae \\
Amoebozoa & Mycetozoa \\
Amoebozoa & Entamoebidae \\
Ciliophora & Tetrahymenidae \\
Echinodermata & Euechinoidea \\
Euglenida & \\
Fungi & Saccharomycetes \\
Fungi & Saccharomycetes \\
Fungi & Saccharomycetes \\
Fungi & Saccharomycetes \\
Heterolobosea & Vahlkampfiidae \\
Hydrozoa & \\
Mesozoa & Rhombozoa \\
Nematoda & Rhabditidae \\
Nematoda & Tylenchina \\
Nematoda & Haemonchidae \\
Nematoda & Trichinellidae \\
Placozoa & \\
Platyhelminthes & Geoplanoidea \\
Platyhelminthes & Schistosomatidae \\
Tunicata & Enterogona \\
& \\
\multicolumn{1}{c}{$\%$} & $1.40 \%$ \\
&
\end{tabular}

Non-specific higher taxonomic grouping Polychaeta $\quad$ segmented worm Polychaeta Tubificina

Acari

Acari

Acari

Arachnida

Araneae
Family

Blattidae

Ectobiidae

Biphyllidae

Cerambicidae

Cerambicidae

Chrysomelidae

Chrysomelidae

Chrysomelidae

Chrysomelidae

Chrysomelidae

Chrysomelidae

Chrysomelidae

Chrysomelidae

Coccinellidae

Coccinellidae

Coccinellidae

Coccinellidae

Coccinellidae

Coccinellidae

Coccinellidae

Coccinellidae

Coccinellidae

Curculionidae

Curculionidae

Curculionidae

Curculionidae

Curculionidae

Curculionidae

Curculionidae

Dermestidae

Dytiscidae

Elateridae

Elateridae

Georissidae

Histeridae

Meloidae

Mycetophagidae

Scarabaeidae

Scarabaeidae
Order total Percent

$1648 \quad 49.08 \%$ 


\begin{tabular}{|c|c|c|c|c|c|c|c|c|c|c|c|}
\hline * & Lepeophtheirus & 3 & Arthropoda & Copepoda & Caligidae (fish lice) & Trypoxylus & 1 & Coleoptera & Scarabaeidae & & \\
\hline & Tachypleus & 1 & Arthropoda & Limulidae & horseshoe crab & * Necrophila & 1 & Coleoptera & Silphidae & & \\
\hline & Branchiostoma & 3 & Cephalochord & a Branchiostomidae & lancelets, amphioxus & Microdera & 1 & Coleoptera & Tenebrionidae & & \\
\hline & Ichthyophthirius & 1 & Ciliophora & Oligohymenophorea & Ophryoglenina & * Tenebrio & 1 & Coleoptera & Tenebrionidae & & \\
\hline * & Saccoglossus & 5 & Eukaryota & Hemichordata & Harrimaniidae & * Tribolium & 1092 & Coleoptera & Tenebrionidae & & \\
\hline & Anoplopoma & 1 & Eukaryota & Vertebrata & Percomorpharia & * Dalmannia & 1 & Diptera & Conopidae & 192 & $5.72 \%$ \\
\hline & Chlamys & 1 & Mollusca & Bivalvia & Pectinidae (scallop) & Муора & 1 & Diptera & Conopidae & & \\
\hline * & Patinopecten & 1 & Mollusca & Bivalvia & Pectinidae (scallop) & * Aedes & 26 & Diptera & Culicidae & & \\
\hline & Bithynia & 4 & Mollusca & Gastropoda & Bithyniidae (snail) & * Anopheles & 31 & Diptera & Culicidae & & \\
\hline & Amphimedon & 1 & Porifera & Niphatidae & sponges & * Culex & 22 & Diptera & Culicidae & & \\
\hline & Suberites & 1 & Porifera & Tetractinomorpha & & Ochlerotatus & 1 & Diptera & Culicidae & & \\
\hline & Rana & 1 & Vertebrata & Amphibia & Ranidae (frogs) & * Teleopsis & 2 & Diptera & Diopsidae & & \\
\hline * & Xenopus & 5 & Vertebrata & Amphibia & Pipidae (frogs) & * Drosophila & 77 & Diptera & Drosophilidae & & \\
\hline & Meleagris & 3 & Vertebrata & Aves & turkey & Musca & 1 & Diptera & Muscidae & & \\
\hline & Taeniopygia & 3 & Vertebrata & Aves & finch & Nemapalpus & 1 & Diptera & Psychodidae & & \\
\hline & Discopyge & 1 & Vertebrata & Chondrichthyes & Narcinidae (ray) & Oxycera & 2 & Diptera & Stratiomyidae & & \\
\hline & Bos & 2 & Vertebrata & Mammalia & Bovidae (cattle) & * Anastrepha & 1 & Diptera & Tephritidae & & \\
\hline & Canis & 2 & Vertebrata & Mammalia & Canidae (dog, wolf) & Bactrocera & 1 & Diptera & Tephritidae & & \\
\hline & Cavia & 2 & Vertebrata & Mammalia & Rodentia (guinea pigs) & * Ceratitis & 24 & Diptera & Tephritidae & & \\
\hline & Ceratotherium & 1 & Vertebrata & Mammalia & Rhinocerotidae (rhinoceros) & Tipula & 1 & Diptera & Tipulidae & & \\
\hline & Dasypus & 1 & Vertebrata & Mammalia & armadillo & Bemisia & 1 & Hemiptera & Aeyrodidae & 98 & $2.92 \%$ \\
\hline & Macaca & 1 & Vertebrata & Mammalia & Primate & * Riptortus & 17 & Hemiptera & Alydidae & & \\
\hline & Monodelphis & 1 & Vertebrata & Mammalia & Didelphidae (oppossum) & * Toxoptera & 2 & Hemiptera & Aphididae & & \\
\hline * & Mus & 2 & Vertebrata & Mammalia & Rodentia (mouse) & * Acyrthosiphon & 60 & Hemiptera & Aphidide & & \\
\hline & Nomascus & 2 & Vertebrata & Mammalia & Primate (gibbon) & Aphis & 1 & Hemiptera & Aphidide & & \\
\hline & Ochotona & 1 & Vertebrata & Mammalia & pika & Laodelphax & 2 & Hemiptera & Delphacidae & & \\
\hline & Odobenus & 5 & Vertebrata & Mammalia & Odobenidae (walrus) & * Oncopeltus & 1 & Hemiptera & Lygaeidae & & \\
\hline & Orcinus & 1 & Vertebrata & Mammalia & Delphinidae (dolphins) & * Lygus & 9 & Hemiptera & Miridae & & \\
\hline & Ornithorhynchus & 7 & Vertebrata & Mammalia & Monotremata (platypus) & Buenoa & 1 & Hemiptera & Notonectidae & & \\
\hline & Oryctolagus & 1 & Vertebrata & Mammalia & Leporidae (rabbits) & * Maconellicoccus & 3 & Hemiptera & Pseudococcidae & & \\
\hline & Otolemur & 2 & Vertebrata & Mammalia & Primate & Diaphorina & 1 & Hemiptera & Psyllidae & & \\
\hline & Ovis & 1 & Vertebrata & Mammalia & sheep & * Apis & 221 & Hymenoptera & Apidae & 548 & $16.32 \%$ \\
\hline & Rattus & 2 & Vertebrata & Mammalia & Rodentia (rat) & * Bombus & 92 & Hymenoptera & Apidae & & \\
\hline & Sarcophilus & 3 & Vertebrata & Mammalia & Dasyuridae (Tasmanian devil) & * Ascogaster & 1 & Hymenoptera & Braconidae & & \\
\hline & Trichechus & 4 & Vertebrata & Mammalia & Trichechidae (manatee) & * Glyptapanteles & 3 & Hymenoptera & Braconidae & & \\
\hline & Anolis & 3 & Vertebrata & Sauria & Iguanidae & * Lysiphlebus & 8 & Hymenoptera & Braconidae & & \\
\hline & Chrysemys & 1 & Vertebrata & Sauria & Testudines & * Nasonia & 66 & Hymenoptera & Braconidae & & \\
\hline & Gallus & 1 & Vertebrata & Sauria & Phasianidae (fowl) & Camponotus & 1 & Hymenoptera & Formicidae & & \\
\hline & Anguilla & 2 & Vertebrata & Teleostei (bony fishes) & Anguillidae (eel) & * Megachile & 79 & Hymenoptera & Megachilidae & & \\
\hline & Anoplopoma & 1 & Vertebrata & Teleostei (bony fishes) & sablefish & * Osmia & 77 & Hymenoptera & Megachilidae & & \\
\hline & Cottus & 1 & Vertebrata & Teleostei (bony fishes) & Cottidae (sculpins) & * Bombyx & 46 & Lepidoptera & Bombycidae & 113 & $3.37 \%$ \\
\hline & Danio & 2 & Vertebrata & Teleostei (bony fishes) & Cyprinidae & * Ostrinia & 6 & Lepidoptera & Crambidae & & \\
\hline & Maylandia & 2 & Vertebrata & Teleostei (bony fishes) & Cichlidae & * Promalactis & 1 & Lepidoptera & Elachistidae & & \\
\hline & Nothobranchius & 5 & Vertebrata & Teleostei (bony fishes) & killifish & * Palaeomicra & 1 & Lepidoptera & Micropterigidae & & \\
\hline * & Salmo & 1 & Vertebrata & Teleostei (bony fishes) & Salmonidae (salmon) & * Helicoverpa & 3 & Lepidoptera & Noctuidae & & \\
\hline & Sinocyclocheilus & 3 & Vertebrata & Teleostei (bony fishes) & Cyprinidae & Spodoptera & 25 & Lepidoptera & Noctuidae & & \\
\hline & Takifugu & 4 & Vertebrata & Teleostei (bony fishes) & Tetraodontidae (puffers) & Euphydryas & 2 & Lepidoptera & Nymphalidae & & \\
\hline & Tetraodon & 1 & Vertebrata & Teleostei (bony fishes) & Tetraodontidae (puffers) & $*$ Heliconius & 11 & Lepidoptera & Nymphalidae & & \\
\hline & Cucumis & 1 & Plant & Cucurbitaceae & melons & Poladryas & 1 & Lepidoptera & Nymphalidae & & \\
\hline & Jatropha & 1 & Plant & Euphorbiaceae (spurges) & & * Papilio & 8 & Lepidoptera & Papilionidae & & \\
\hline$*$ & Psychopsis & 1 & Plant & Orchidacea & orchid & Antheraea & 1 & Lepidoptera & Saturniidae & & \\
\hline & & & & & & Manduca & 1 & Lepidoptera & Sphingidae & & \\
\hline & Total & 122 & & $\%$ non-insect metazoans: & $3.63 \%$ & * Monochamus & 3 & Lepidoptera & Sphingidae & & \\
\hline & & & & & & Neococytius & 1 & Lepidoptera & Sphingidae & & \\
\hline & (continued to right) & & & & & * Edosa & 1 & Lepidoptera & Tineidae & & \\
\hline & & & & & & Leucomele & 1 & Lepidoptera & Tineidae & & \\
\hline & & & & & & * Tinea & 1 & Lepidoptera & Tineidae & & \\
\hline & & & & & & * Protohermes & 1 & Megaloptera & Corydalidae & & \\
\hline & & & & & & * Chrysopa & 434 & Neuroptera & Chrysopidae & 434 & $12.92 \%$ \\
\hline & & & & & & * Locusta & 1 & Orthoptera & Acrididae & & \\
\hline & & & & & & * Schistocerca & 2 & Orthoptera & Acrididae & & \\
\hline & & & & & & Dianemobius & 1 & Orthoptera & Gryllidae & & \\
\hline & & & & & & * Gryllus & 4 & Orthoptera & Gryllidae & & \\
\hline & & & & & & * Pediculus & 145 & Pthiraptera & Pediculidae & 145 & $4.32 \%$ \\
\hline & & & & & & Xenopsylla & 1 & Siphonaptera & Pulicidae & & \\
\hline & & & & & & Dipseudopsis & 1 & Trichoptera & Dipseudopsidae & 13 & $0.39 \%$ \\
\hline & & & & & & Total & 3191 & & & 3191 & $94.74 \%$ \\
\hline
\end{tabular}

* indicates a difference in presence (present in one sample but not the other) or a difference in quantity of transcripts found.

$\dagger$ Non-insect taxa are highly variable, so generally recognizable taxonomic names are listed. Taxonomic identifications are from NCBI Taxonomy Browser, http://www.ncbi.nlm.nih.gov/Taxonomy/Browser/wwwtax.cgi

Metazoans are included in the "microbial" portion of the spreadsheet (ie. fungi); those categorized in \% microbial may be associated with symbionts or food, rather than insect. 
Table 2. Selected sequences (16) from treatment samples that appear identical, and their actual nt identities. Abbreviations incorporated into sequence IDs: Insect egg (IE); pollen.

\begin{tabular}{|c|c|c|c|c|c|c|c|c|c|}
\hline \multicolumn{10}{|l|}{ Tribolium sequence pairs: } \\
\hline Sequence ID & length $\mathrm{gi}$ & Bit Score & Expect & Sequence ID & length & $\begin{array}{l}\text { gi and description (RNA) } \\
\text { gi|189233647|ref|XM_967463.2| PREDICTED: Tribolium }\end{array}$ & Bit Score & Expect & nt Identities \\
\hline IEcomp8421_c0_seq1 & 2902 gi|189233647|ref| & 258 & $8 \quad 3 E-64$ & Pcomp8751_c0_seq4 & 2917 & 7 castaneum similar to beta-arrestin 1 (LOC661293), mRNA & 258 & $8 \quad 3 E-64$ & $62902 / 2902,100 \%$ \\
\hline IEcomp6954_c0_seq1 & 3236 gi|189233777|ref| & 163 & $1 \mathrm{E}-35$ & Pcomp8809_c0_seq1 & 3268 & $\begin{array}{l}\text { gi|189233777|ref|XM_970744.2| PREDICTED: Tribolium } \\
8 \text { castaneum similar to Mitf CG17469-PB (LOC659688), mRNA }\end{array}$ & 163 & 3 1E-35 & 3 3192/3192, 100\% \\
\hline IEcomp7095_c0_seq1 & 6235 gi|189233793|ref| & 93.7 & $2 E-14$ & |Pcomp3394_c0_seq1 & 6173 & $\begin{array}{l}\text { gi|189233793|ref|XM_001814833.1| PREDICTED: Tribolium } \\
\text { 3 castaneum similar to Gb1-cadherin (LOC661970), mRNA } \\
\text { gi|189233859|ref|XM_967562.2| PREDICTED: Tribolium } \\
\text { castaneum similar to succinyl-coa synthetase beta chain }\end{array}$ & 93.7 & $7 \quad 2 \mathrm{E}-14$ & $46096 / 6174,99 \%$ \\
\hline IEcomp14202_c0_seq1 & 1597 gi|189233859|ref| & 87.7 & $3 E-13$ & Pcomp14830_c0_seq1 & 1591 & 1 (LOC661403), mRNA & 87.7 & $7 \quad 3 \mathrm{E}-13$ & $131584 / 1586,99 \%$ \\
\hline IEcomp10244_c0_seq3 & 2101 gi|189233868|ref| & 214 & $3 E-51$ & |Pcomp6797_c0_seq2 & 2098 & $\begin{array}{l}\text { gi|189233868|ref|XM_963541.2| PREDICTED: Tribolium } \\
8 \text { castaneum similar to AGAP007416-PA (LOC657053), mRNA } \\
\text { gi|189233948|ref|XM_001815112.1| PREDICTED: Tribolium } \\
\text { castaneum similar to CG11526 CG11526-PA (LOC661304), }\end{array}$ & 214 & $4 \quad 3 E-51$ & $92096 / 2096,100 \%$ \\
\hline IEcomp12989_c0_seq1 & 2756 gi|189233948|ref| & 157 & $7 E-34$ & Pcomp13520_c0_seq1 & 2727 & $\begin{array}{l}7 \text { mRNA } \\
\text { gi|189234001|ref|XM_967366.2| PREDICTED: Tribolium } \\
\text { castaneum similar to CG10249 CG10249-PD (LOC661190), }\end{array}$ & 157 & $7 \quad 7 E-34$ & $162703 / 2720,99 \%$ \\
\hline IEcomp2432_c0_seq1 & 3559 gi|189234001|ref| & 91.7 & $4 \mathrm{E}-14$ & Pcomp7193_c0_seq2 & & $\begin{array}{l}2 \text { mRNA } \\
\text { gi|189234377|ref|XM_001815988.1| PREDICTED: Tribolium } \\
\text { castaneum similar to PCl domain containing } 2 \text { (LOC100142368), }\end{array}$ & 91.7 & $7 \quad 4 \mathrm{E}-14$ & $13559 / 3559,100 \%$ \\
\hline IEcomp2367_c0_seq1 & 1500 gi|189234377|ref| & 139 & 9E-29 & Pcomp21956_c0_seq1 & & $\begin{array}{l}7 \text { mRNA } \\
\text { gi|189234523|ref|XM_967649.2| PREDICTED: Tribolium } \\
\text { castaneum similar to zinc finger protein, putative (LOC661494), }\end{array}$ & 139 & 9 9E-29 & $151494 / 1494,100 \%$ \\
\hline IEcomp22006_c0_seq1 & 1683 gi|189234523|ref| & 83.8 & $5 E-12$ & |Pcomp5895_c0_seq1 & 1612 & 2 mRNA & 83.8 & $85 \mathrm{E}-12$ & 14 1609/1609, 100\% \\
\hline \\
\hline Sequence ID & length gi (per analysis) & Bit Score & & Sequence ID & length & $\begin{array}{l}\text { updated similarity (protein)^ } \\
\text { gi91085467 XP_970131.1 } 340 \text { aa PREDICTED: guanine }\end{array}$ & Bit Score & Expect & \\
\hline IEcomp9964_c0_seq1 & 3242 gi|332375734|gb| & 347 & 4E-91 & Pcomp9907_c0_seq1 & 3227 & $\begin{array}{l}7 \text { nucleotide-binding protein subunit beta-1 } \\
\text { gi91087239 XP_975511.1 PREDICTED: eukaryotic initiation }\end{array}$ & 347 & $7 \quad 4 \mathrm{E}-91$ & $83224 / 3227,99 \%$ \\
\hline IEcomp9135_c0_seq1 & $1474 \mathrm{gi}|459305579| \mathrm{gb} \mid$ & 490 & O 1E-134 & Pcomp9568_c0_seq1 & 1476 & $\begin{array}{l}6 \text { factor 4A-III [Tribolium castaneum] } 404 \text { aa } \\
\text { gi189241344 PREDICTED: obg-like ATPase } 1 \text { [Tribolium }\end{array}$ & 490 & 0 1E-134 & $71462 / 1465,99 \%$ \\
\hline IEcomp5768_c0_seq1 & $1484 \mathrm{gi}|459307330| \mathrm{gb} \mid$ & 151 & $2 E-32$ & |Pcomp13212_c0_seq2 & 1479 & $\begin{array}{l}9 \text { castaneum] Sequence ID: ref|XP_969865.2|Length: } 399 \\
\text { gi91088003 XP_973796.1 PREDICTED: putative RNA-binding }\end{array}$ & 151 & $12 \mathrm{E}-32$ & $21472 / 1479,99 \%$ \\
\hline IEcomp11734_c0_seq2 & 1416 gi|459311962|gb| & 210 & O $3 E-50$ & Pcomp11908_c0_seq4 & 1376 & $\begin{array}{l}6 \text { protein Luc7-like } 2 \text { [Tribolium } \\
\text { gi91080555 XP_967130.1 PREDICTED: clathrin light chain }\end{array}$ & 210 & $0 \quad 3 E-50$ & 11 1369/1378, 99\% \\
\hline IEcomp14296_c0_seq1 & $1161 \mathrm{gi}|459314268| \mathrm{gb} \mid$ & 81.8 & $1 \mathrm{E}-11$ & |Pcomp14860_c0_seq1 & 1186 & $\begin{array}{l}6 \text { isoform X1 [Tribolium castaneum]. } \\
\text { gi288869512 NP_001165863.1 XP_970587 ribosomal protein S3 }\end{array}$ & 81.8 & $8 \quad 1 \mathrm{E}-11$ & $181161 / 1161,100 \%$ \\
\hline IEcomp13735_c0_seq1 & $838 \mathrm{gi}|459323274| \mathrm{gb} \mid$ & 125 & $7 E-25$ & Pcomp7175_c0_seq1 & 847 & $\begin{array}{l}7 \text { [Tribolium castaneum]. } \\
\text { gi642911142 XP_008200598.1 PREDICTED: protein disulfide. }\end{array}$ & 125 & $5 \quad 7 E-25$ & $17836 / 837,99 \%$ \\
\hline IEcomp14748_c0_seq1 & $1270 \mathrm{gi}|459324930| \mathrm{gb} \mid$ & 91.7 & $2 \mathrm{E}-14$ & |Pcomp15141_c0_seq1 & 1286 & 6 isomerase A6 [Tribolium castaneum]. & 91.7 & $7 \quad 2 E-14$ & 19 1265/1265, 100\% \\
\hline
\end{tabular}

analyzed by BLASTx and the closest annotated description is provided. Bit Scores and Expect values refer to the tBLASTx result, listed in the "gi (per analysis)" column.

Table 3. Longest 15 sequences unique in treatments. P: pollen diet, IE: insect egg diet.

\begin{tabular}{|c|c|c|c|c|c|c|}
\hline & $\begin{array}{l}\text { Unique sequences in pollen } \\
\text { diet sample }\end{array}$ & & Putative transcript type & $\begin{array}{c}\text { Unique sequence in insect } \\
\text { egg diet sample }\end{array}$ & & Putative transcript type \\
\hline & Sequence ID & length & & Sequence ID & length & \\
\hline 1 & Pcomp7841_c0_seq1 & 2080 & Glycoside hydrolase & IEcomp11484_c0_seq1 & 2167 & uncharacterized hypothetical protein \\
\hline 2 & Pcomp12927_c0_seq2 & 1918 & Synaptic vesicle protein & IEcomp13279_c0_seq1 & 1935 & uncharacterized hypothetical protein \\
\hline 3 & Pcomp7836_c0_seq1 & 1683 & Fatty acid binding protein & IEcomp4164_c0_seq1 & 1910 & $\begin{array}{l}\text { putative transposon } \\
\end{array}$ \\
\hline 4 & Pcomp49463_c0_seq1 & 1645 & Reverse transcriptase & IEcomp13213_c0_seq1 & 1889 & unknown \\
\hline 5 & Pcomp529_c0_seq1 & 1146 & 7 transmembrane receptor & IEcomp11074_c0_seq1 & 1775 & unknown \\
\hline 6 & Pcomp26787_c0_seq1 & 1144 & unidentifiable & IEcomp11853_c0_seq1 & 1607 & unknown \\
\hline 7 & Pcomp38644_c0_seq1 & 1049 & organic anion transporter & IEcomp6564_c0_seq1 & 1583 & Ankyrin repeat protein \\
\hline 8 & Pcomp39179_c0_seq1 & 982 & Apolipophorin precursor & IEcomp9557_c0_seq1 & 1575 & unknown \\
\hline 9 & Pcomp57928_c0_seq1 & 982 & unknown & IEcomp4213_c0_seq1 & 1450 & Lygus lineolaris virus 1 \\
\hline 10 & Pcomp6831_c0_seq1 & 975 & uncharacterized protein & IEcomp13459_c0_seq21 & 1332 & Drosophila A virus structural protein \\
\hline 11 & Pcomp33_c0_seq1 & 964 & Synaptic vesicle protein & IEcomp13259_c0_seq4 & 1276 & RhoGAP15B \\
\hline 12 & Pcomp9099_c0_seq1 & 959 & unknown & IEcomp35607_c0_seq1 & 1091 & copia-type polyprotein \\
\hline 13 & Pcomp59166_c0_seq1 & 956 & Glycoside hydrolase & IEcomp2540_c0_seq2 & 1078 & HARBI1-like nuclease \\
\hline 14 & Pcomp57181_c0_seq1 & 947 & transposable element-like & IEcomp27681_c0_seq1 & 1016 & unidentifiable \\
\hline 15 & Pcomp4200_c0_seq1 & 828 & unidentifiable & IEcomp31819_c0_seq1 & 1016 & Cytochrome P450 18A1 \\
\hline
\end{tabular}

The fifteen longest unique sequences from each diet treatment are listed in Table 3. While the unique sequences in pollen-fed transcripts appear to be related to carbohydrate cleavage, which seems logical for animals digesting plant materials, the unique sequences from the egg-fed did not appear to match any obvious category of transcript, other than the insect virus [18] mentioned earlier.

Further analyses of the transcripts reported here will provide insight on genes that are linked to differential metabolism of plant and animal based diets. The sequences identified here, after validation among further representatives of this species, will be used to measure quantitative changes in gene expression between insects utilizing multiple sources of foods, and those that are deprived of specific dietary components. Potential projects include varying diet at different stages of insect development; this species is known to utilize different diet components at different stages of development [19-21]. Another possibility is to evaluate genetic responses to specific prey, as $C$. maculata has been shown to utilize prey of specific sizes and species [22]. This will help us understand how to produce high quality generalist predator bio- 
logical control agents, and assure conservation of beneficial insects in our constantly changing world environment. The details of the genes expressed by this omnivorous insect, and their correlation with diet and nutrition, will provide insights into nutritional health of other omnivores including humans.

\section{Methods}

\section{Insect culture}

Lady beetles used to establish laboratory colonies were collected from fields surrounding the USDA-ARS and Mississippi State University Delta Research and Extension Center in Stoneville, MS, 38776. Insect cultures were maintained in the National Biological Control Laboratory at Stoneville, MS without wild specimen introgression from August 2010 through the time of RNA sample collection in March 2013. Insects were reared as larvae in Petri dishes of sizes ranging from 35 to $250 \mathrm{~mm}$ with mesh glued into one side of the dish for ventilation, and as adults in $5.25 \times 5.25$ inch cages [12]. Temperature was generally maintained at $24^{\circ} \mathrm{C}$ for 16 lighted hours and 19. C for 8 dark hours in Percival (Perry, IA) E30B growth chambers. Larvae and adults were fed ad libitum a combination of pollen, Daphnia, Brewer's yeast, honey, and eggs of laboratory cultures of Lygus spp. [12]. Water was provided in $1.5 \mathrm{ml}$ microcentrifuge tubes with caps removed and plugged with cotton.

\section{Inbreeding for homozygosity}

Inbreeding of beetle stock was performed by isolating individual gravid females from the primary wild type colony, collecting and rearing all eggs from individual females, and continuing culture using only offspring of the most fertile and fecund female. From the resulting culture the inbreeding step (female selection) was repeated for a total of six isofemale (I6) selection steps.

\section{Sample preparation}

Insect specimens from a single egg mass collected from the I6 colony were fed standard diet as larvae. Surviving individuals were isolated after pupation and provided either pollen alone or Lygus eggs alone upon adult eclosion. Total RNA was isolated from whole individual adults six days after adult eclosion. Specimens were spray washed with reverse osmosis (RO) water to remove any food particles. After one hour isolation (resting from wash and away from food) specimens were briefly anaesthetized with carbon dioxide. Insects were transferred to sample tubes and crushed whole in RNA extraction buffer using blue Kontes ${ }^{\circledR}$ (Kimble Chase, Thermo Fisher
Scientific, Waltham, MA) pestle. Total RNA was extracted using USB PrepEase total RNA kit following manufacturer instructions (Affymetrix, Santa Clara, CA). Samples were measured using a NanoDrop 1000 (Thermo Fisher Scientific) spectrophotometer, and the samples with highest final concentration and highest 260/230 ratio were chosen for sequencing. RNA samples were kept in ultralow freezer set for $-75^{\circ} \mathrm{C}$ until shipping.

\section{Transcript Sequencing}

Total RNA samples were shipped on dry ice to the University of Washington High-Throughput Genomics Center, WTC East, Suite 600, 2211 Elliott Ave., Seattle, WA 98121-1692. Illumina (Illumina, Inc., San Diego, CA, USA) RNA-seq library construction, $36 \mathrm{bp}$ single end multiplex quality control library testing, and 76 bp paired end multiplex $4 x$ sequencing was followed by Trinity (Broad Institute, MA) contiguous sequence assembly [23]. Sequences were assembled by diet treatment (eggs only or pollen only) and as a combined group assembly. Assembled sequences were limited by request to >200nt. Assembled sequences were provided to the USDA ARS Genomics and Bioinformatics Research Unit (GBRU) in Stoneville, MS for further analysis.

\section{Sequence Analyses}

Assembled sequences were analyzed by NCBI BLAST ${ }^{\circledR}$ (US National Library of Medicine) [16] using the tBLASTx "search translated nucleotide database using a translated nucleotide query" (database accessed 22 May 2013). BLAST results were analyzed by diet treatment (eggs only or pollen only) and as a combined group. Spreadsheet results were sorted by Hit Score and only those sequences in the two treatment groups meeting the combined criteria of $>500$ nucleotide (nt) and E (expect) score $<\mathrm{E}-10$ were used for further analysis.

Because the sequenced samples were derived from two individual insects that differed only in their adult diet, the transcriptomes were expected to be nearly identical for those sequences representing genes that were not associated with diet. To verify that assemblies represented genetically similar individuals as expected, sixteen sequences were chosen based on apparent identity by length of assembled sequence and closest tBLASTx hit. Sequences were chosen from the group most similar to Tribolium castaneum sequences and from the group most similar to Dendroctonus ponderosae sequences, with the assumption that sequences from other beetles would represent conserved transcripts. Sequences from pollen-fed 
$(\mathrm{P})$ and insect egg-fed (IE) were compared at the nucleotide level using BLAST.

\section{Acknowledgements}

I acknowledge the many Biological Science Technicians who supported this work: Catherine Louise Smith, Fannie Mae Byrd, Ebony Williams, Mary Elizabeth Huddleston, and Joseph Grey Ballenger. Many thanks go to Dr. Jeffrey Gore of Mississippi State University for collecting and donating live lady beetle specimens, and to Drs. Don Weber and Jonathan Lundgren of USDA ARS for helpful advice on raising beetles in the laboratory. Insect eggs (Lygus spp.) were kindly provided by the NBCL Insect Production team, Dr. M. Guadalupe Rojas, Orswald (Archie) Clark, Derrick T. Jones, Matthew D. McDaniel, and Terry E. Johnson. Assistance in ordering sequencing from University of Washington High-Throughput Genomics Center was provided by Dr. Peter Sabo and other helpful support staff. Batch BLAST analyses were performed by Linda Ballard, Bioinformaticist of the ARS Genomics and Bioinformatics Research Unit led by Dr. Brian Scheffler. Mention of trade names or commercial products in this article is solely for the purpose of providing specific information and does not imply recommendation or endorsement by the US Department of Agriculture (USDA). USDA Agricultural Research Service is an equal opportunity employer. I thank Mark Weaver and Jeremy Marshall for reviewing an earlier version the manuscript prior to submission.

\section{Competing Interests}

The author has declared that no competing interest exists.

\section{References}

1. Gordon RD: The Coccinellidae (Coleoptera) of America north of Mexico. J New York Entomol S. 1985;93:1-912.

2. Pérez OG, Hoy MA: Reproductive incompatibility between two subspecies of Coleomegilla maculata (Coleoptera: Coccinellidae). Fla Entomol. 2002;85(1):203-207.

3. Halloy SRP, Barratt BIP: Patterns of abundance and morphology as indicators of ecosystem status: A meta-analysis. Ecol Complex. 2007;4(3):128-147.

4. Lundgren JG, Weber DC: Changes in digestive rate of a predatory beetle over its larval stage: implications for dietary breadth. J Insect Physiol. 2010;56(4):431-437.

5. Pilorget L, Buckner J, Lundgren JG: Sterol limitation in a pollen-fed omnivorous lady beetle (Coleoptera: Coccinellidae). J Insect Physiol. 2010;56(1):81-87.

6. Lundgren JG, Wiedenmann RN: Nutritional suitability of corn pollen for the predator Coleomegilla maculata (Coleoptera: Coccinellidae). J Insect Physiol. 2004;50(6):567-575.

7. Michaud JP, Grant AK: Suitability of pollen sources for the development and reproduction of Coleomegilla maculata (Coleoptera: Coccinellidae) under simulated drought conditions. Biol Control. 2005;32(3):363-370.

8. Phoofolo MW, Obrycki JJ: Potential for intraguild predation and competition among predatory Coccinellidae and Chrysopidae. Entomol Exp Appl. 1998;89(1):47-55.
9. Vargas G, Michaud JP, Nechols JR: Maternal effects shape dynamic trajectories of reproductive allocation in the ladybird Coleomegilla maculata. Bull Entomol Res. 2012;102(5):558-565.

10. Michaud JP. Coccinellids in Biological Control. In: Hodek I, van Emden HF, and Honek A, ed. Ecology and behaviour of the ladybird beetles (Coccinellidae). West Sussex: Wiley-Blackwell; 2012: 488-519.

11. Kuwayama H, Gotoh H, Konishi Y, Nishikawa H, Yaginuma T, Niimi T. Establishment of Transgenic Lines for Jumpstarter Method Using a Composite Transposon Vector in the Ladybird Beetle, Harmonia axyridis. PLoS One. 2014;9(6):e10080.

12. Allen ML, Riddick EW: A system for harvesting eggs from the pink-spotted lady beetle. Psyche 2012;Article923653.

13. Allen ML, Ballenger JG: Genetics and Characteristics of a Pigmentation Defective Laboratory Strain of the Lady Beetle, Coleomegilla maculata. Adv in Entomol. 2014;2:161-166.

14. Gregory TR, Nedved O, Adamowicz SJ. C-value estimates for 31 species of ladybird beetles (Coleoptera: Coccinellidae). Hereditas. 2003;139(2):121-7.

15. Dolezel J, Bartos J, Voglmayr H, Greilhuber J: Nuclear DNA content and genome size of trout and human. Cytom Part A. 2003;51(2):127-128.

16. Altschul SF, Madden TL, Schaffer AA, Zhang J, Zhang Z, Miller W, Lipman DJ: Gapped BLAST and PSI-BLAST: a new generation of protein database search programs. Nucleic Acids Res. 1997;25(17):3389-3402.

17. Hoy MA, Yu F, Meyer JM, Tarazona OA, Jeyaprakash A, Wu K: Transcriptome sequencing and annotation of the predatory mite Metaseiulus occidentalis (Acari: Phytoseiidae): a cautionary tale about possible contamination by prey sequences. Exp Appl Acarol. 2013;59(3):283-296

18. Perera OP, Snodgrass GL, Allen KC, Jackson RE, Becnel JJ, O'Leary PF, Luttrell RG: The complete genome sequence of a single-stranded RNA virus from the tarnished plant bug, Lygus lineolaris (Palisot de Beauvois). J Invertebr Pathol. 2012;109(1):11-19.

19. Matos B, Obrycki JJ: Prey suitability of Galerucella calmariensis L. (Coleoptera: Chrysomelidae) and Myzus lythri (Schrank) (Homoptera: Aphididae) for development of three predatory species. Environ Entomol. 2006;35(2):345-350.

20. Wiebe AP, Obrycki JJ: Prey suitability of Galerucella pusilla eggs for two generalist predators, Coleomegilla maculata and Chrysoperla carnea. Biol Control. 2002;23(2):143-148.

21. Riddick EW, Wu Z, Rojas MG: Is Tetranychus urticae suitable prey for development and reproduction of naïve Coleomegilla maculata? Insect Sci. 2014;21(1):99-104.

22. Roger C, Coderre D, Boivin G: Differential prey utilization by the generalist predator Coleomegilla maculata lengi according to prey size and species. Entomol Exp Appl. 2000;94(1):3-13.

23. Grabherr MG, Haas BJ, Yassour M, Levin JZ, Thompson DA, Amit I, Adiconis X, Fan L, Raychowdhury R, Zeng $Q$ et al: Full-length transcriptome assembly from RNA-Seq data without a reference genome. Nat Biotech. 2011;29(7):644-652. 